\title{
Survey of a Body Composition Selection and its Association with Heart Rate Product in Junior Boys and Girls Systolic Blood Pressure
}

\author{
Masoud Mabani ${ }^{1}$, Mehdi Abbaszadegan ${ }^{2 *}$ and Mahrokh Dehghani ${ }^{3}$ \\ ${ }^{1}$ Master of Science in Exercise Physiology \\ ${ }^{2}$ Department of Sport sciences, Payame Noor University, Iran \\ ${ }^{3}$ Department of sport sciences, University of Mohaghegh Ardabili, Iran \\ *Corresponding author: Mehdi Abbaszadegan, Department of Sport sciences, Iran
}

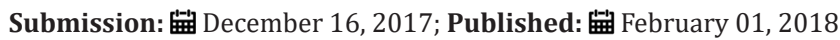

\begin{abstract}
Approach of this study was explaining of relationship and comparison of body composition characteristics with heart rate product in junior boys and girls systolic blood pressure. The statistical population of this study was 200 junior girls and boys with an age range of 13-15 years old. After completing the full explanation of the purpose and method of performing the research for the selected subjects, we received written consent from them and the medical questionnaire was provided to them. Heart rate and systolic blood pressure were measured at rest time. The findings showed that there is a correlation between body mass, body mass index and waist to hip measure ratio with heart rate product in systolic blood pressure ( $\mathrm{P}<0.05)$. Body mass index had the highest relationship with heart rate product in systolic blood pressure. The effect of body fat mass and body mass index on heart rate product was different in systolic blood pressure in both sexes. The interactive effect of gender on any of the body fat mass and body mass index variables was similar to systolic blood pressure. It can be concluded that the factors of iconology and body composition (height, body weight, waist to hip measure ratio, body mass index) have a significant role in the changes in the value of the heart rate in the systolic blood pressure.
\end{abstract}

Keywords: Physical composition; Heart rate product; Systolic blood pressure; Body mass index; Junior girls and boys

\section{Introduction}

Studies conducted over the past decade showed that body composition is an important factor in the health of the person and the profile of his physical and mental fitness [1]. For this reason, measuring body composition at the moment is commonplace in many health and sports centers. One of the main reasons for the prevalence of measuring body composition is the relationship that exists between many factors of body composition, especially body fat percentage, with physical and mental illnesses. In the daily life of mankind, there are many people who are physically inadequate. And perhaps most of them are not even aware of their abnormal condition and even do not care about it. Overweight and obesity can be mentioned here [2]. Many researchers have estimated the measurements of metering indicators as the easiest way to assess obesity and the risk of cardiovascular diseases [3]. The best indicators of health determinants are localized subcutaneous fat, body mass index, fat mass and body type. The heart is a vital pump that needs a good supply of oxygen and nutrients to do its job well. If these sources are inadequate, they will cause heart failure. When conducting heavy and prolonged physical activity such as aerobic exercise, heart rate and systolic blood pressure are considered as two main determinants. When the severity and duration of these two factors increases, it is an indication of cardiac output and more cardiac activity. In this case, the heart needs more oxygen and more nutrients. Because the heart is a highly oxidative member that has a fairly high oxygen consumption. The heart needs to consume more oxygen to recover the energy. Therefore, myocardial metabolism and its function are completely interrelated. Recognizing the differences in body composition and physiological composition and their effects on myocardial metabolism may be a sign of understanding the differences observed in the prevalence of cardiovascular disorders and diseases [4]. Heart rate product in systolic blood pressure (RPP) is a computational and indirect estimation of oxygen consumption in the heart. This technique can easily be used in sports tests and cardiovascular patients. Any overall value of the heart rate product in more than 10,000 systolic pressure indicates an increased risk of heart disease [5]. Body composition subsets such as body mass index are variables that changes with age. Their assessments are used to estimate health levels and determine excess weight and obesity [6]. Research has shown that body mass index (BMI) has a significant relationship with the concentration of dangerous fats and systolic and diastolic 
blood pressure in both genders [7]. Awareness of anthropometric changes, body composition and body mass index at childhood and adolescence can evaluate their nutritional status, development and maturity, and provide a picture of the health status of the community in the future [8]. Most researches have focused on the effect of physical activity on RPP, and have less attention to its relationship with body composition. Researches that conducted on the body composition have focused on its relationship with blood pressure. In this research, we tried to evaluate the effect of selected compounds from body composition with heart rate product in systolic blood pressure of junior boys and girls.

\section{Research Methodology}

This research was conducted to describe and explain the relationship between body composition and heart rate product in junior boys and girls systolic blood pressure. The research method is semi-experimental, applied and in-field type. The research design is descriptive and correlational. The statistical population of this study was junior girls and boys with a range of 13-15 years old in the city of Miandoab. The community consists of four middle schools with 800 students, 200 of them were randomly selected for this research. After completing the full explanation of the purpose and method of performing the research for the selected subjects, we received written consent from them and medical questionnaire was provided to them. Systolic and diastolic blood pressure and resting heart rate were measured by Microlife digital barometer, a German manufactured instrument with product no of BP A130 and serial number of 4719003310707.

\section{Body mass}

The amount of matter that presents in the body called mass. The body mass of the subjects in this study was measured with a medical scale and with a $1 \%$ error in a kilogram [3]. The multiplication of heart rate (s/min) and systolic blood pressure (RPP) is called mercury in millimeters and is considered as the best indirect way to measure adequate oxygen consumption in the heart and determines some kind of heart rate [9].

Rate Pressure Product $(\mathrm{RPP})=$ Resting Heart Rate $(\mathrm{RHR}) *$ Systolic Blood Pressure (SBP)

Mass and body height of the subjects were measured using a medical scale and digital medical clock in kilograms and centimeters respectively and recorded in the corresponding form.

\section{Research Operation Method}

The height of the subjects was measured with a measuring device in the form of standing and without shoes in centimeter and recorded in standard form. The body mass of the subjects was measured without shoes, with simple and light sportswear, and using a digital medical scale in kilogram. Height and weight measurements were used to calculate body mass index (BMI). An automatic device was used to measure systolic pressure and heart rate in subjects. The digital barometer has the capability to simultaneously report pulse rates, systolic blood pressure and diastolic blood pressure. To ensure the validity and reliability of the device, measurements were taken three times at intervals of at least three minutes from five participants. To measurement, the mercury barometer was tied on the right arm and the digital barometer was tied on the left hand arm. The mean recorded values of the two devices in nine times measurement was less than a millimeter of mercury. The reliability of the digital barometer was determined on the basis of conducted operations. There was no significant difference in pulse rate and systolic and diastolic blood pressure reported by the device in three measurements. The strength of the device was to report the figure of variables on the screen, which eliminates the measurement error in the digits and takes little time to measure. Each subject, after a short rest, sat on the chair in a quiet environment with a short sleeve dress, placing the left arm on the table with palm up. The lower edge of the armband (cuff) was about 2.5 centimeters higher than the subject's elbow cavity. By pressing the start button, the blood pressure measurement began, and at the end of measurement the systolic pressure and subjects' heart rate was recorded in millimeters of mercury, and the number per minute noted on the screen. This measurement was repeated at least twice within 10 minutes, and if the difference in systolic blood pressure was five $\mathrm{mm} \mathrm{Hg}$ and less, the mean of both was calculated and recorded.

\section{Statistical Method}

First, the normality status of the data (mean and standard deviation) was evaluated using Kolmogorov-Smirnov and Levine tests; then, changes in each of the indicators during different stages of measurement were investigated using descriptive statistics and Pearson correlation coefficient and Two-way variance analysis tests. All operations and statistical analyzes were performed at a significant level of 5\% using SPSS version 22 and Excel 2013 software.

\section{Research Findings}

Table 1: Mean and standard deviation of physiological and anthropometric characteristics of subjects.

\begin{tabular}{|c|c|c|c|c|c|c|}
\hline Indicators & Age (year) & Weight (Kg) & Height (m) & BMI (Kg/m²) & $\begin{array}{c}\text { Systolic Blood Pressure } \\
\text { (mmHg) }\end{array}$ & $\begin{array}{c}\text { Diastolic Blood } \\
\text { Pressure (mmHg) }\end{array}$ \\
\hline Mean \pm SD & $13.67 \pm 0.81$ & $59.16 \pm 17.04$ & $163.53 \pm 1361$ & $27.66 \pm 3.57$ & $68.24 \pm 14.59$ & 109.78 .23 .98 \\
\hline
\end{tabular}

Table 1 shows the mean and standard deviation of individual characteristics (age, weight, height, body mass index ...).

According to the obtained data, they are close to each other in all physiological factors. The rate of association between selected body composition and heart rate product in systolic blood pressure of junior girls and boys is presented in Table 2 .

According to Table 2, Body mass variables ( $\mathrm{P}=0.005)$, Body mass index $(\mathrm{P}=0.0001)$ and waist to hip ratio $(\mathrm{P}=0.004)$ have a significant relationship with heart rate product in Systolic blood pressure. 
Table 2: The rate of association between selected body composition and heart rate product in systolic blood pressure (200 persons).

\begin{tabular}{|c|c|c|}
\hline \multirow{2}{*}{ Variables } & \multicolumn{2}{|c|}{ Heart Rate Product in Systolic Blood Pressure } \\
\hline \multirow{2}{*}{ Body mass } & r Pearson & $0.02^{*}$ \\
\cline { 2 - 3 } & significance level & 0.005 \\
\hline \multirow{2}{*}{ BMI } & r Pearson & $0.28^{*}$ \\
\cline { 2 - 3 } & significance level & 0.001 \\
\hline \multirow{2}{*}{ Waist to hip ratio } & r Pearson & 0.2 \\
\cline { 2 - 3 } & significance level & 0.004 \\
\hline
\end{tabular}

*A significant level of $5 \% \quad{ }^{* *}$ A significant level at $1 \%$

The results of two-way ANOVA for the selected effect of physical compositions on the heart rate product in systolic blood pressure in junior girls and boys are shown in Table 3 .

According to Table 3, gender $(\mathrm{P}=0.24)$, waist to hip ratio index $(\mathrm{P}=0.08)$, gender and body mass $(\mathrm{P}=0.57)$, waist to hip ratio index and gender $(\mathrm{P}=0.14)$ simultaneously did not have a significant effect on heart rate product in systolic blood pressure, but body weight $(\mathrm{P}=0.01)$, $\mathrm{BMI}(\mathrm{P}=0.0001)$, and $\mathrm{BMI}$ and gender $(\mathrm{P}=0.0001)$ simultaneously have a significant effect on the product of heart rate in systolic blood pressure.

Table 3: Test of selected physical compositions effects on Heart rate product in Systolic blood pressure.

\begin{tabular}{|c|c|c|c|c|}
\hline & Degree of Freedom & Average Square & F Statistics & Significance Level \\
\hline Gender & 1 & 13765311 & 1.33 & 0.24 \\
\hline Body mass & 48 & 17526056 & $1.7^{*}$ & 0.01 \\
\hline Gender* body mass & 22 & 9436910 & 0.91 & 0.0001 \\
\hline BMI & 103 & 17583696 & $5.53^{* *}$ & 0.001 \\
\hline Gender* BMI & 17 & 12404332 & 1.43 & 0.08 \\
\hline WHR & 28 & 16075496 & 1.41 & 0.14 \\
\hline Gender* WHR & 15 & 15803282 & & \\
\hline
\end{tabular}

*A significant level of $5 \% \quad{ }^{* *} \mathrm{~A}$ significant level at $1 \%$

\section{Discussion and Conclusion}

The findings showed that the mean BMI $\left(33.21 \mathrm{~kg} / \mathrm{m}^{2}\right)$, body mass $(15.59 \mathrm{~kg})$, waist to hip ratio index $(82.00 \mathrm{~kg})$, and have a relation with mean heart rate product in systolic blood pressure (4.101894). It can be concluded that the increase in each of the selected subscales of selected physical compositions may increase the rate of heart rate product in the systolic blood pressure in subjects. The resting heart rate of girls aged 13 to 15 years old was $94.18 \pm 14.78$, diastolic blood pressure was $63.39 \pm 7.99$ and systolic blood pressure was $108.65 \pm 12.08$ and for boys aged $13-15$ years for boys the resting heart rate is $103.2 \pm 21.81$, diastolic blood pressure is $72.24 \pm 16.76$ and the systolic blood pressure is $118.35 \pm 16.19$ respectively. Rational, geographical and lifestyle factors may affect the range of heart and blood pressure ranges. Considering that the range of heart rate changes is much higher than the range of systolic blood pressure changes, it may be more than 10,000 of the value of heart rate in blood pressure in this study is due to the high heart rate of subjects, which is a significant number of them were under the age of ten. More research is needed to reject or accept this theory. The results of this study are similar to the results of research by Mota et al., Zhang et al., Lewis et al., Michelle et al., sim et al., Jackson et al. Zhang et al (2012) found that systolic and diastolic blood pressure in both genders had a positive correlation with fat mass, lean body mass, and BMI, which is consistent with the result of the present study. The findings of this study showed that BMI in girls and boys was directly related to heart rate product in systolic blood pressure [10]. In a study on preschool children, Luis (2011) concluded that a one-unit increase in BMI was associated with an increase of about $0.57 \mathrm{mmHg}$ in systolic blood pressure. This data shows a strong correlation between systolic blood pressure and BMI, which is consistent with the results of the present study, considering the effect of systolic blood pressure on the heart rate product in systolic blood pressure. The results of this study showed that body mass index (BMI) has a significant relationship with heart rate product in systolic blood pressure [11]. Michelle (2011) concluded that age, height, weight, waist to hip ratio index and BMI had a significant correlation with heart rate, blood pressure, and heart rate product in systolic blood pressure, which consistent with present research results. The present study indicates a positive correlation between weight, BMI and height with changes in heart rate product in systolic blood pressure [12].

Mohan (2005) and Nielsen (2002) in their study on teenager girls and boys aged 14 to 18 evaluated the relationship between BMI, heart rate, systolic blood pressure, diastolic blood pressure 
and heart rate product in systolic blood pressure [2,3]. The results of this study showed that there is a positive correlation between BMI and heart rate product in systolic blood pressure in both genders. Also, the results of Mohan's research showed that there is a relationship between gender and BMI and blood pressure which associated with body metabolism. The present study showed that BMI affects heart rate product in systolic blood pressure changes in both genders. According to the results of this study and its comparison with other studies, it can be concluded that the anthropometric factors and composition of body (height, body mass, waist to hip ratio, BMI) have a significant role in the changes in numerical value of the heart rate product in systolic blood pressure. The results showed that the increase in any of the body composition subscales probably increases the numerical value of the heart rate product in systolic blood pressure and thus increases the risk of cardiovascular disease risk.

\section{References}

1. Mannucci E, Ricca V, Barciulli E, Di Bernardo M, Travaglini R, et al. (1999) Quality of life and overweight: the obesity related well-being (Orwell 97) questionnaire. Addictive behaviors 24(3): 345-357.

2. Ravisankar P, Udupa K, Prakash ES (2005) Correlation between body mass index and blood pressure indices, handgrip strength and handgrip endurance in underweight, normal weight and overweight adolescents. Indian J Physiol Pharmacol 49(4): 455-461.

3. Hu G, Barengo NC, Tuomilehto J, Lakka TA, Nissinen A, et al. (2004) Relationship of physical activity and body mass index to the risk of hypertension: a prospective study in Finland. Hypertension 43(1): 2530.

4. Bagali S (2012) Aging and gender effects on rate-pressure product: An index of myocardial oxygen consumption. International Journal of Biomedical and Advance Research 3(3): 175-178.

5. Understanding Exercise Intensity and rate pressure product (RPP).

6. Nass R, Thorner M (2002) Impact of the GH-cortisol ratio on the agedependent changes in body composition. Growth Horm IGF Res 12(3): 147-161.

7. Hills AP, King NA, Byrne NME (2007) Children, obesity and exercise: prevention, treatment and management of childhood and adolescent obesity. Routledge.

8. Vincent SD, Pangrazi RP, Raustorp A, Tomson LM, Cuddihy TF (2003) Activity levels and body mass index of children in the United States, Sweden, and Australia. Med Sci Sports Exerc 35(8): 1367-1373.

9. Mota J, Soares-Miranda L, Silva JM, Dos Santos SS, Vale S (2012) Influence of body fat and level of physical activity on rate-pressure product at rest in preschool children. Am J Hum Biol 24(5): 661-665.

10. Zhang YX, Wang SR (2011) Relation of body mass index, fat mass index and fat-free mass index to blood pressure in children aged 7-12 in Shandong, China. Ann Hum Biol 38(3): 313-316.

11. Gopinath B, Baur LA, Garnett S, Pfund N, Burlutsky G, et al. (2011) Body mass index and waist circumference are associated with blood pressure in preschool-aged children. Ann Epidemiol 21(5): 351-357.

12. Abiodun AG, Egwu MO, Adedoyin RA (2011) Anthropometric indices associated with variation in cardiovascular parameters among primary school pupils in ile-ife. International journal of hypertension 2011(2011): Article ID 186194. 